Pacific Journal of Mathematic 


\title{
LOCAL CONNECTEDNESS IN DEVELOPABLE SPACES
}

\author{
HAROLD W. MARTiN
}

\begin{abstract}
A space is spherically connected if and only if it has an admissible semi-metric $d$ such that $d$-spheres of radius less than one are connected. It is shown that a developable space is locally connected if and only if it is spherically connected. A semi-metric space is $K$-semi-metrizable if and only if it admits a semi-metric $d$ such that $d(A, B)>0$ whenever $A$ and $B$ are disjoint compact sets. It is shown that in the class of locally connected rim compact spaces, the $K$-semimetrizable spaces are precisely the developable $\gamma$-spaces. An example is given of a locally connected, locally compact $K$ semi-metrizable Moore space which is not metrizable.
\end{abstract}

1. Introduction. A topological space is said to be rim compact provided that each point has a local basis of open sets which have compact boundaries. A space is locally connected provided that each point of the space has a local basis of connected open sets. If $R$ is the set of all rational points of the plane $E^{2}$, the $E^{2}-R$ is an example of a locally connected, rim compact space which is nowhere locally compact.

If $d$ is a semi-metric for a space $X$, then $d$ is said to be a $K$ semi-metric provided that $d(A, B)>0$ whenever $A$ and $B$ are disjoint compact subsets of $X$. It seems to be unknown whether every regular semi-metrizable space, ${ }^{1}$ or even developable space, ${ }^{2}$ has a compatible $K$-semi-metric. We define a topological space $X$ to be $d$-spherically connected provided that $X$ has a compatible semi-metric $d$ such that every $d$-sphere $S_{d}(x, e)=\{y: d(x, y)<e\}$ of radius less than one is connected. A space is said to be spherically connected provided that it is $d$-spherically connected for some compatible semi-metric $d$.

Theorem 5.2 of [3] may be phrased as follows: let $X$ be a rim compact space; if $X$ is $d$-spherically connected by virtue of a $K$-semimetric $d$, then $X$ is metrizable. Also, P. Zenor has shown that a locally connected rim compact space is metrizable if and only if it

${ }^{1}$ A space $X$ is semi-metrizable provided there exists a nonnegative, real-valued function $d$ on $X \times X$, called a semi-metric, which satisfies the following three conditions: (i) $d(x, y)=d(y, x)$; (ii) $d(x, y)=0$ iff $x=y$; (iii) for $x$ in $X$ and $A \subset X$, we have $x \in \operatorname{cl}(A)$ iff $d(d(x, A))=\inf \{x, a): a \in A\}=0$.

${ }^{2}$ A sequence $G_{1}, G_{2}, \cdots$ of open covers of a space $X$ is called a development provided that $\left\{S t\left(x, G_{n}\right): n \in Z^{+}\right\}$is a local base at $x$ for each $x$ in $X$. A space is developable provided it has a development. A regular developable space is called a Moore space. 
has a regular $G_{i}$-diagonal ${ }^{3}$ [6]. A comparison of these two theorems suggests the question of whether local connectedness and spherical connectedness are equivalent concepts in the class of semi-metrizable spaces. The purpose of this note is to give a partial answer to this question by showing that local connectedness and spherical connectedness are equivalent in the class of developable spaces. Heath's theorem also suggests the question of whether a rim compact, spherically connected space is metrizable. We answer this question negatively by showing that there exists a locally compact, locally connected, completely regular Moore space $X$ which is $K$-semi-metrizable but not metrizable. This same example shows that Zenor's theorem cannot be improved by replacing "regular $G_{\delta}$-diagonal" by " $G_{\delta}^{*}$-diagonal". We shall also show that a locally connected rim compact space is $K$-semi-metrizable if and only if it is a developable $\gamma$-space. ${ }^{5}$

\section{Theorems and example.}

THEOREM 1. Every spherically connected space is locally connected; every locally connected developable space is spherically connected.

Proof. Let $X$ be a $d$-spherically connected space. The space $X$ is locally connected provided that each component of each open set is open. Let $G$ be an arbitrary open subset of $X$ and let $C$ be an arbitrary component of the subspace $G$. Given $x \in C$, there exists $e>0$ such that $e<1$ and $S_{d}(x, e) \subset G$. Since $S_{d}(x, e)$ is connected, we have $S_{d}(x, e) \subset C$. It follows that $x \in \operatorname{int}(C)$, that is, that $C$ is an open set, whence $X$ is locally connected.

To prove the second part of Theorem 1 , let $X$ now denote a locally connected developable space. Let $V_{1}, V_{2}, \cdots$, be a development for $X$. Since $X$ is a developable space, $X$ has a compatible semimetric $p$ such that if $x \in X$ and $e>0$ is given, then $x$ has a neighborhood $V$ such that $p(a, b)<e$ for every $a, b \in V$ [1, page 128]. For each $x \in X$ and natural number $n$, let $d_{n}(x)$ denote a connected open neighborhood of $x$ such that $p(a, b)<1 / n$ for all $a, b \in d_{n}(x)$ and such that $d_{n}(x) \subset v$ for some $v \in V_{n}$; furthermore, choose the sets $d_{n}(x)$ so

\footnotetext{
${ }^{3}$ A space $X$ has a regular $G_{\delta}$-diagonal provided $X$ has a sequence $G_{1}, G_{2} \cdots$ of open covers such that if $x$ and $y$ are distinct points of $X$, then $x$ has a neighborhood $V$ for which $y \notin c l\left(S t\left(V, G_{n}\right)\right)$ for some natural number $n$.

${ }^{4}$ A space $X$ has a $G_{\delta}^{*}$-diagonal if $X$ has a sequence $G_{1}, G_{2}, \cdots$ of open covers such that if $x$ and $y$ are distinct points of $X$, then $y \notin c l\left(\operatorname{St}\left(x, G_{n}\right)\right)$ for some natural number $n$.

${ }^{5}$ A space $X$ is a $\gamma$-space iff there exists a function $g$ from $Z^{+} \times X$ into the open sets of $X$ such that: (i) $\left\{g(n, x): n \in Z^{+}\right)$is a local base at $x$ with $g(n+1, x) \subset g(n, x)$ and (ii) if $A \subset G$ where $A$ is compact and $G$ open, then there exists $n \in Z^{+}$such that $g(n, a) \subset G$ for every $a \in A$.
} 
that $d_{n+1}(x) \subset d_{n}(x)$ for all $n$. Let $G_{n}=\left\{d_{n}(x): x \in X\right\}$; then, $G_{1}, G_{2}$, $\cdots$, is a development for $X$. For $x \in X$ and $n=1,2, \cdots$, set $g_{n}(x)=$ $S t\left(x, G_{n}\right)$. If $x \in g_{n}\left(x_{n}\right)$ for $n \in Z^{+}$, then there exists a sequence $\left\{y_{n}\right\}$ in $X$ such that $x, x_{n} \in d_{n}\left(y_{n}\right)$, whence $p\left(x, x_{n}\right)<1 / n$, that is, $\left\{x_{n}\right\}$ converges to $x$. Note also that $d_{n+1}(x) \subset d_{n}(x)$ implies that $g_{n+1}(x) \subset g_{n}(x)$. Consequently, we may define a semi-metric $d$ for $X$, which is equivalent to $p$, in the following standard way [3, Theorem 3.2]: if $x \neq y$, let $d(x, y)=1 / n$ where $n$ is the least natural number $k$ such that $x \notin$ $g_{k}(y)$ and $y \notin g_{k}(x)$.

Let $x \in X$ and $0<e<1$. The proof will be completed by showing that $S_{d}(x, e)$ is connected. Let $n$ be the particular natural number which satisfies the relations $1 /(n+1)<e \leqq 1 / n$. Observe that $S_{d}(x$, $e)=S_{d}(x, 1 / n)$. Let $y \in S_{d}(x, e)$; then $d(x, y)<1 / n$, so that $x \in g_{n}(y)$ or $y \in g_{n}(x)$. There must exist a set $S_{y} \in G_{n}$ with $x, y \in S_{y}$. If $z \in S_{y}$, then $x \in S t\left(z, G_{n}\right)=g_{n}(z)$, so that $d(x, z)<1 / n$, that is, $z \in S_{d}(x, e)$; consequently, $S_{y} \subset S_{d}(x, e)$. It follows that $S_{d}(x, e)=\bigcup\left\{S_{y}: y \in S_{d}(x, e)\right\}$; but then $S_{d}(X, e)$ is connected since each set $S_{y}$ is connected and contains the common point $x$, completing the proof.

In the proof of Theorem 1 , we have $p(x, y) \leqq d(x, y)$ for all $x$, $y \in X$. Then, $p$ is a $K$-semi-metric implies that $d$ is a $K$-semi-metric. But, Zenor has shown that a developable space $X$ has a regular $G_{\delta^{-}}$ diagonal if and only if $X$ has a $K$-semimetric $p$ such that if $x \in X$ and $e>0$ is given, then $x$ has a neighborhood $V$ such that $p(a, b)<e$ whenever $a, b \in V,[7]$. These remarks, together with the proof of Theorem 1, imply the following:

COROLLARY 2. If $X$ is a locally connected developable space which has a regular $G_{b}$-diagonal, then $X$ has a $K$-semi-metric $d$ such that $X$ is $d$-spherically connected.

Corollary 2 suggests the question of whether a locally connected, $K$-semi-metrizable developable space has a $K$-semi-metric $d$ such that the space is $d$-spherically connected. This question is important since an affirmative answer, in conjunction with Theorem 5.2 of [3], would imply that every $K$-semi-metrizable, locally connected, rim compact space is metrizable. This, however, is not the case, as shown by the following example, which is a variation of the space $\sum_{B}$ of [5, page 376], of a locally compact, locally connected, nonmetrizable, $K$ semi-metrizable, quasi-metric ${ }^{6}$ Moore space.

Given a quasi-metric space $(X, d)$ we let $T_{d}$ denote the topology

${ }^{6} \quad$ A space $X$ is said to be quasi-metrizable provided that there exists a nonnegative real-valued function $d$ on $X \times X$, called a quasi-metric, which satisfies the following conditions: (i) $d(x, y)=0$ iff $x=y$; (ii) $d(x, y) \leqq d(x, z)+d(z, y)$; (iii) the collection $\left\{S_{d}(x, e): x \in X\right.$ and $e>0\}$ forms a base for the topology for $X$, where $S_{d}(x, e)=\{y \in X: d(x, y)<e\}$. 
having sets of the form $\{y: d(x, y)<1 / n\}$ as an open basis, where $x$ ranges over the set $X$ and $n \in Z^{+}=\{1,2, \cdots\}$.

ExAmPle 3. Let $P=\{(x, y\}: 0<x<1$ and $0<y \leqq 1\}$. If $x$ is a real number and $0<a<\pi$, then let $R(x, a)$ denote the ray, emanating from the point $(x, 0)$ in the plane, which lies in the upper half plane and makes an angle of a radians with the positive direction along the $x$-axis. Let $R=\{R(x, a): 0<x<1$ and $0<a<\pi\}$ and let $X=P \cup R$.

Let $I=\{(x, 0): 0 \leqq x \leqq 1\}$ and let $p$ be a fixed point of $I$. Let $x$ and $y$ be elements of $P$. Define $d_{p}(x, y)=|| x-p|-| y-p||+a$ where $|p-q|$ is the usual Euclidean distance between points $p$ and $q$ of the plane and $a$ is the positive angle, in radians, between the line segment joining $p$ to $x$ and the line segment joining $p$ to $y$. Now define $d(x, y)=\sup \left\{d_{p}(x, y): p \in I\right\}$.

If $y \in P$ and $R(x, a) \in R$, let $d(y, R(x, a))=2 \pi$ and let $d(R(x, a)$, $y)=|(x, 0)-y|+b$ where $b$ is the positive angle, in radians, between the ray $R(x, a)$ and the line segment joining $(x, 0)$ to $y$.

If $R(x, a)$ and $R(y, b)$ are elements of $R$, define $d(R(x, a), R(y, b))=$ $2 \pi$ if $x \neq y$ and $d(R(x, a), R(y, b))=|a-b|$ if $x=y$.

It is straightforward to show that $d$ is a quasi-metric for the set $X$. The topological space $\left(X, T_{d}\right)$ is a nonnormal, locally compact, locally connected, completely regular, quasi-metrizable Moore space.

If $R(x, a) \in R$, define $g_{n}(R(x, a))$ as follows: $R(y, b) \in g_{n}(R(x, a))$ if and only if $x=y$ and $|a-b|<1 / n$, and $(p, q) \in P$ belongs to $g_{n}(R(x$, $a)$ ) if and only if $\sqrt{(x-p)^{2}+q^{2}}<1 / n$ and $|a-c|<1 / n$ where $c$ is the angle in radians between the ray $R(x, a)$ and the line segment connecting the points $(p, q)$ and $(x, 0)$. The topology $T_{d}$ has the collections $\left\{g_{n}(R(x, a)): n \in Z^{+}\right\}$as local open bases for points $R(x, a)$ in $R$ and the points of $P$ have their usual neighborhood systems. We shall now construct a compatible $K$-semi-metric for the space $\left(X, T_{d}\right)$.

Let $y$ and $z$ denote distinct points of $P$ and let $I$ be defined as above. Let $L$ denote the line $y=1$ in the plane. If $x \in I$ and $p \in$ $P$, let $\pi_{x}(p)$ be the point of intersection of the line $L$ with the line connecting the points $\mathrm{x}$ and $p$. Now define $\delta(y, z)=\max \left\{\mid \pi_{x}(y)-\right.$ $\left.\pi_{x}(z) \mid: x \in I\right\}$. If $p \in P$ and $R(x, a) \in R$, define $\delta(p, R(x, a))=\mid p-(x$, $0) \mid+b$ where $b$ is the angle, in radians, between the ray $R(x, a)$ and the ray emanating from $(x, 0)$ and containing the point $p$. Finally, if $R(x, a)$ and $R(y, b)$ are points of $R$ with $x \neq y$, let $\delta(R(x, a)$, $R(y, b))=1$ and let $\delta(R(x, a), R(x, b))=|a-b|$. It is easy to show that $\delta$ is a compatible semi-metric for the space $\left(X, T_{d}\right)$. It remains only to show that $\delta$ is a $K$-semi-metric. Note that $\delta$ is a $K$-semimetric for the space $X$ if and only if whenever $\delta\left(w_{n}, z_{n}\right) \rightarrow 0, w_{n} \rightarrow$ 
$w$ and $z_{n} \rightarrow z$, then $w=z$. With respect to this criterion for $K$-semimetrizability, the only nonobvious case is that in which $w=R(x, a)$, $z=R(x, b)$ and the sequences $\left\{w_{n}\right\}$ and $\left\{z_{n}\right\}$ are in the set $P$. Therefore, suppose that $w_{n} \rightarrow R(x, a)$ and $z_{n} \rightarrow R(x, b)$ where $w_{n}, z_{n} \in P$ for all $n$ and $a \neq b$. Let $p(a)$ be the point of intersection of the line $L$ and the ray $R(x, a)$ and likewise let $p(b)$ be the point of intesection of $L$ with the ray $R(x, b)$. Since $w_{n} \rightarrow R(x, a)$, we must have $\pi_{(x, 0)}\left(w_{n}\right) \rightarrow$ $p(a)$ along the line $L$; similarly, $\pi_{(x, 0)}\left(z_{n}\right) \rightarrow p(b)$ along the line $L$. Since $\delta\left(w_{n}, z_{n}\right)=\max \left\{\left|\pi_{y}\left(w_{n}\right)-\pi_{y}\left(z_{n}\right)\right|: y \in I\right\}$, we have

$$
\left|\pi_{(x, 0)}\left(w_{n}\right)-\pi_{(x, 0)}\left(z_{n}\right)\right| \leqq \delta\left(w_{n}, z_{n}\right) .
$$

It follows readily that $\delta\left(w_{n}, z_{n}\right)>1 / 2(|p(a)-p(b)|)$ for sufficiently large values of $n$, completing the proof that $\delta$ is a $K$-semi-metric.

The space of Example 3 is $K$-semi-metrizable and spherically connected, but not $d$-spherically connected whenever $d$ is a $K$-semi-metric for $X$.

In the class of locally connected, rim compact spaces, we have the following coincidence theorem.

THEOREM 4. A locally connected, rim compact space is K-semimetrizable if and only if it is a developable $\gamma$-space.

Proof. It is easy to show that a developable $\gamma$-space is $K$-semimetrizable and we therefore omit the details. Let $d$ be a compatible $K$-semi-metric for a locally connected, rim compact space $X$. We may choose a system $\left\{g(n, x): x \in X ; n \in Z^{+}\right\}$of open, connected subsets of $X$ such that the following two conditions hold: (i) $\left\{g(n, x): n \in Z^{+}\right\}$ is a local base at $x$ with $g(n+1, x) \subset g(n, x)$; (ii) if $y \in g(n, x)$, then $d(x, y)<1 / n$. Note that if $C$ and $D$ are disjoint compact subsets of $X$, then there exists a natural number $n$ such that $g(n, C) \cap D=\varnothing$, where $g(n, C)=\bigcup\{g(n, c): c \in C)$.

Let $A$ be an arbitrary compact subset of $X$ and $n$ be a natural number. Choose a finite number of points in $A$, say $x_{1}, x_{2}, \cdots, x_{m}$, such that $A \subset \bigcup\left\{g\left(n, x_{i}\right): i=1,2, \cdots, m\right\}$. Let $A_{i}=A \cap g\left(n, x_{i}\right)$; the sets $g\left(n, A_{i}\right)$ are connected for $1 \leqq i \leqq m$.

We are now in a position to show that $X$ is a $\gamma$-space. Let $K$ be a compact subset of $X$ and let $W$ be an open set containing $K$. Choose a finite number of open sets $G_{1}, G_{2}, \cdots, G_{p}$ such that for each $i=1,2, \cdots, p$, the set $B d\left(G_{i}\right)$ is compact, $G_{i} \subset W$, and $K \subset \bigcup\left\{G_{i}\right.$ : $i=1,2, \cdots, p\}=G$. Since $B d(G) \subset \bigcup\left\{B d\left(G_{i}\right): i=1,2, \cdots, p\right\}$, we have that $B d(G)$ is compact. Then, $K \cap B d(G)=\varnothing$ and there exists a natural number $n$ such that $g(n, K) \cap B d(G)=\varnothing$. By the preceding paragraph, we have $K=K_{1} \cup \cdots \cup K_{m}$ for some natural number $m$, where $g\left(n, K_{i}\right)$ 
is connected for $1 \leqq i \leqq m$. Since $K_{i} \subset K \subset G$ and $g\left(n, K_{i}\right)$ is connected, and since $g\left(n, K_{i}\right) \cap B d(G)=\varnothing$, we must have $g\left(n, K_{i}\right) \subset G$ for $1 \leqq i \leqq m$. Note that $g(n, K)=\bigcup\left\{g\left(n, K_{i}\right): 1 \leqq i \leqq m\right\}$. It follows that $g(n, K) \subset G \subset W$, completing the proof that $X$ is a $\gamma$-space. It is easy to show that a semi-metrizable $\gamma$-space is developable, e.g., use Theorem 3.3 of [3], and the proof of Theorem 4 is complete.

3. Open questions. In [2], Fletcher and Lindgren conjectured that every quasi-metrizable space admits a compatible non-Archimedean quasi-metric. Y. A. Kofner gave a counterexample to this conjecture [4]; however, Kofner's space is not developable, so the FletcherLindgren conjecture remains open for developable spaces. Recall that a developable space admits a compatible non-Archimedean quasi-metric if and only if it is orthocompact [2]. We therefore ask:

QUESTION 1. Is the space $X$ of Example 3 orthocompact?

Question 2 is motivated by Theorem 1 and Question 3 by Theorem 4 and other theorems in the literature.

QUESTION 2. Is every locally connected, semi-metrizable space necessarily spherically connected?

QUESTION 3. Is every regular semi-metrizable space necessarily $K$-semi-metrizable? If not, is every Moore space a $K$-semi-metrizable space?

\section{REFERENCES}

1. A. V. Arhangel'skî̂, Mappings and spaces, Uspehi Mat. Nauk, 21, No. 4 (130) 133$184=$ Russian Math. Surveys, 21 (1966), No. 4, 115-162.

2. P. Fletcher and W. F. Lindgren, Orthocompactness and strong Čech completeness in Moore spaces, Duke Math. J., 39 (1972), 753-766.

3. R. W. Heath, Arcwise connectedness in semi-metric spaces, Pacific J. Math., 12 (1962), 1301-1319.

4. Ya. A. Kofner, On 4-metrizable spaces, Mat. Zametki, 13, No. 2, (1973), 277-287.

5. R. L. Moore, Foundations of Point Set Theory, Amer. Math. Soc. Coll. Publ., vol. 13, New York, (1962), revised edition.

6. P. L. Zenor, Spaces with regular $C_{\delta}$-diagonals, Proc. Third Prague Top. Symp., (1971), 471-473.

7. P. L. Zenor, On spaces with regular Gs-diagonals, Pacific J. Math., 40 (1972), 759763.

Received March 12, 1975 and in revised form August 17, 1975.

TEXAS TeCH UNIVERSITY

Current address: College of St. Scholastica Duluth, Minnesota 55811. 


\section{PACIFIC JOURNAL OF MATHEMATICS}

\section{EDITORS}

RICHARD ARENS (Managing Editor) University of California

Los Angeles, California 90024

\section{R. A. BEAUMONT}

University of Washington Seattle, Washington 98105
J. DugundjI Department of Mathematics University of Southern Californı Los Angeles, California 90007

D. Gilbarg AND J. Milgram Stanford University Stanford, California 94305

\section{ASSOCIATE EDITORS}

E. F. BECKENBACH

B. H. NeUMANN

F. WOLF

K. YosHidA

\section{SUPPORTING INSTITUTIONS}

UNIVERSITY OF BRITISH COLUMBIA CALIFORNIA INSTITUTE OF TECHNOLOGY UNIVERSITY OF CALIFORNIA MONTANA STATE UNIVERSITY UNIVERSITY OF NEVADA NEW MEXICO STATE UNIVERSITY OREGON STATE UNIVERSITY UNIVERSITY OF OREGON OSAKA UNIVERSITY

\author{
UNIVERSITY OF SOUTHERN CALIFORNIA \\ STANFORD UNIVERSITY \\ UNIVERSITY OF TOKYO \\ UNIVERSITY OF UTAH \\ WASHINGTON STATE UNIVERSITY \\ UNIVERSITY OF WASHINGTON \\ AMERICAN MATHEMATICAL SOCIETY \\ NAVAL WEAPONS CENTER
}




\section{Pacific Journal of Mathematics}

\section{Vol. 61, No. $1 \quad$ November, 1975}

Jiří Adámek, V. Koubek and Věra Trnková, Sums of Boolean spaces represent every

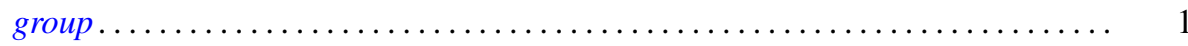

Richard Neal Ball, Full convex l-subgroups and the existence of $a^{*}$-closures of

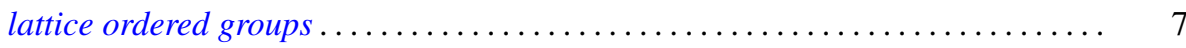

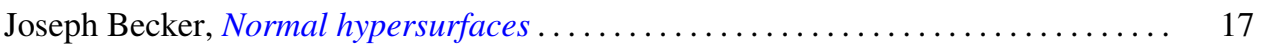

Gerald A. Beer, Starshaped sets and the Hausdorff metric . . . . . . . . . . . . . 21

Dennis Dale Berkey and Alan Cecil Lazer, Linear differential systems with

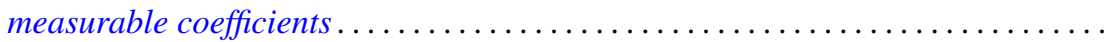

Harald Boehme, Glättungen von Abbildungen 3-dimensionaler

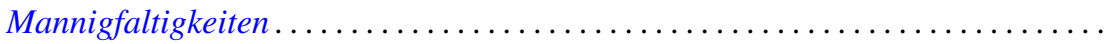

Stephen LaVern Campbell, Linear operators for which $T^{*} T$ and $T+T^{*}$

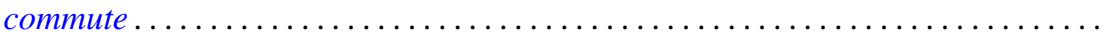

H. P. Dikshit and Arun Kumar, Absolute summability of Fourier series with

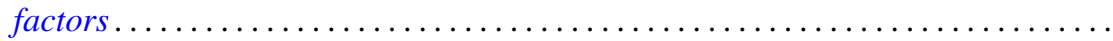

Andrew George Earnest and John Sollion Hsia, Spinor norms of local integral

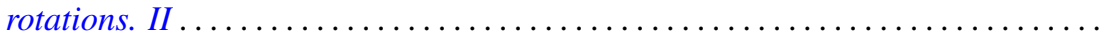

Erik Maurice Ellentuck, Semigroups, Horn sentences and isolic structures .........

Ingrid Fotino, Generalized convolution ring of arithmetic functions . . . . . . . . . . .

Michael Randy Gabel, Lower bounds on the stable range of polynomial rings .......

Fergus John Gaines, Kato-Taussky-Wielandt commutator relations and

characteristic curves

Theodore William Gamelin, The polynomial hulls of certain subsets of $C^{2}$

R. J. Gazik and Darrell Conley Kent, Coarse uniform convergence spaces. . .

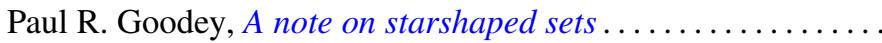

Eloise A. Hamann, On power-invariance

M. Jayachandran and M. Rajagopalan, Scattered compactification for $N \cup\{P\}$. . .

V. Karunakaran, Certain classes of regular univalent functions .

John Cronan Kieffer, A ratio limit theorem for a strongly subadditive set function in a locally compact amenable group .................

Siu Kwong Lo and Harald G. Niederreiter, Banach-Buck measure, density, and uniform distribution in rings of algebraic integers ........

Harold W. Martin, Contractibility of topological spaces onto metric spaces ....

Harold W. Martin, Local connectedness in developable spaces .

A. Meir and John W. Moon, Relations between packing and covering numbers of a tree.

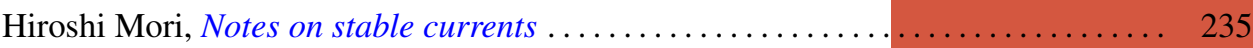

Donald J. Newman and I. J. Schoenberg, Splines and the logarithmic function . . . . 241

M. Ann Piech, Locality of the number of particles operator....

Fred Richman, The constructive theory of $K T$-modules .......

Gerard Sierksma, Carathéodory and Helly-numbers of

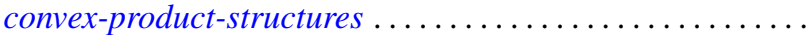

Raymond Earl Smithson, Subcontinuity for multifunctions .... . . 